\title{
HDAC6 inhibition prevents TNF-a-induced caspase 3 activation in lung endothelial cell and maintains cell-cell junctions
}

\author{
Jinyan $\mathbf{Y u}^{1,2,3}$, Mengshi Ma ${ }^{1,2}$, Zhongsen $\mathrm{Ma}^{1}$, Jian $\mathrm{Fu}^{2,3}$ \\ ${ }^{1}$ Department of Respiratory Medicine, The Second Hospital of Jilin University, Changchun, Jilin, P.R. China \\ ${ }^{2}$ Center for Research on Environmental Disease, College of Medicine, University of Kentucky, Lexington, KY, USA \\ ${ }^{3}$ Department of Toxicology and Cancer Biology, College of Medicine, University of Kentucky, Lexington, KY, USA \\ Correspondence to: Jian Fu, email: jian.fu@uky.edu \\ Zhongsen Ma, email: mazhongsen2005@163.com
}

Keywords: caspase, endothelial, HDAC6, barrier function, inflammation

Received: May 05, 2016

Accepted: June 30, 2016

Published: July 13, 2016

\section{ABSTRACT}

Pro-inflammatory mediators such as TNF-a induce caspase activation in endothelial cells, which leads to degradation of cellular proteins, induction of apoptotic signaling, and endothelial cell dysfunction. New therapeutic agents that can inhibit caspase activation may provide protection against inflammatory injury to endothelial cells. In the present study, we examined the effects of selective histone deacetylase 6 (HDAC6) inhibition on TNF-a induced caspase 3 activation and cell-cell junction dysfunction in lung endothelial cells. We also assessed the protective effects of HDAC6 inhibition against lung inflammatory injury in a mouse model of endotoxemia. We demonstrated that selective HDAC6 inhibition or knockdown of HDAC6 expression was able to prevent caspase 3 activation in lung endothelial cells and maintain lung endothelial cell-cell junctions. Mice pre-treated with HDAC6 inhibitors exhibited decreased endotoxin-induced caspase 3 activation and reduced lung vascular injury as indicated by the retention of cell-cell junction protein VE-Cadherin level and alleviated lung edema. Collectively, our data suggest that HDAC6 inhibition is a potent therapeutic strategy against inflammatory injury to endothelial cells.

\section{INTRODUCTION}

Inflammation-mediated endothelial cell damage including inflammatory lung vascular injury is often associated with caspase activation and endothelial cell barrier dysfunction [1]. Over-production of proinflammatory mediators such as tumor necrosis factor (TNF)- $\alpha$ is a major cause of endothelial cell injury during inflammation [1]. Tumor necrosis factor (TNF)- $\alpha$-induced caspase activation and endothelial cell dysfunction contribute to inflammatory vascular injury in endotoxemia and sepsis [1]. New agents that can prevent inflammatory injury to endothelial cells could provide therapeutic benefits.

Activated caspases are known to induce apoptosis by cleaving endogenous substrates and alter other cellular functions including cell-cell junctions [2-4]. Caspase-3 activation plays an important role in endothelial cell barrier dysfunction as a result of apoptotic signaling and re-organization of cell-cell junction proteins including ZO-1 and VE-Cadherin [2-4]. Maintaining of endothelial barrier integrity requires interactions of intercellular junctions, actin cytoskeleton and microtubules [5]. Adherens junctions (AJs) and tight junctions play essential roles in supporting endothelial barrier function [2-4]. ZO-1 and VE-cadherin are main components of TJs and AJs respectively. TNF- $\alpha$ induces endothelial barrier disruption by dis-organization of microtubule, actin cytoskeleton and cell junctions [6-9].

HDAC6, a member of class II histone deacetylase (HDACs) [10-12], is localized in the cytoplasm and the nucleus [10-12]. HDAC6 inhibition possesses antitumor and anti-inflammation effects [13-15]. HDAC6 has many endogenous substrates including chaperones and cytoskeletal proteins [16]. Stabilization of cytoskeletal proteins and modulation of chaperones by HDAC6 may protect against endothelial cell injury [16, 17]. Indeed, HDAC6 deletion renders mice less vulnerable to endotoxin-induced inflammatory injury [18]. However, the effects of HDAC6 inhibition on caspase 3 activation and endothelial cell function remain to be determined. 
Tubastatin A and CAY10603 are potent and highly selective HDAC6 inhibitors with IC50 of $15 \mathrm{nM}$ and $2 \mathrm{pM}$ in a cell-free assay, respectively [19]. Tubastatin A has been reported to possess anti-inflammatory effects [20-23]. Tubastatin A treatment can prevent stress responses and prolong survival during systemic inflammation [24-28].

In the present study, we investigated the effects of HDAC6 knockdown and HDAC6 inhibitors Tubastatin A and CAY 10603 on TNF- $\alpha$-induced caspase 3 activation in endothelial cells in vitro. We examined the effects of Tubastatin A and CAY10603 on cell-cell junction integrity and endothelial permeability in primary human lung endothelial cells. Furthermore, the effects of Tubastatin A and CAY 10603 on caspase 3 activation and lung edema formation in vivo was assessed in a mouse model of endotoxemia.

\section{RESULTS}

\section{Tubstatin a inhibits TNF $\alpha$-induced caspase-3 activation in lung endothelial cells}

TNF- $\alpha$ is a major pro-inflammatory mediator known to induce endothelial injury during inflammation $(17,21,27)$. TNF- $\alpha$ induces caspase 3 activation which leads to subsequent cellular damage including endothelial barrier dysfunction [29, 30]. We first assessed whether HDAC6 inhibition can block caspase-3 activation in lung endothelial cells. HPAECs and HLMVECs were pretreated with Tubstatin A, then challenged with TNF- $\alpha$. Our results showed that Tubstatin A potently blocked TNF- $\alpha$ induced caspase- 3 activation (cleaved caspase-3 level) in endothelial cells (Figure 1).

\section{HDAC6 knockdown and CAY10603 treatment attenuate TNF- $\alpha$-induced caspase- 3 activation in endothelial cells}

To confirm the specific effects of HDAC6 inhibition on TNF- $\alpha$-induced caspase- 3 activation, we used HDAC6 siRNA knockdown and another selective HDAC6 inhibitor CAY10603 in our study. HPAECs were challenged with TNF- $\alpha$ after siRNA knockdown or CAY10603 treatment. HDAC6 knockdown and CAY10603 treatment both inhibited TNF- $\alpha$-induced caspase- 3 activation (Figure 2 ).

\section{HDAC6 inhibition alleviates TNF- $\alpha$-induced disruption of tight junctions in endothelial cells}

Caspase- 3 activation is associated with endothelial cell-cell junction disruption [2-4]. We next conduct experiments to assess whether HDAC6 inhibition could
A

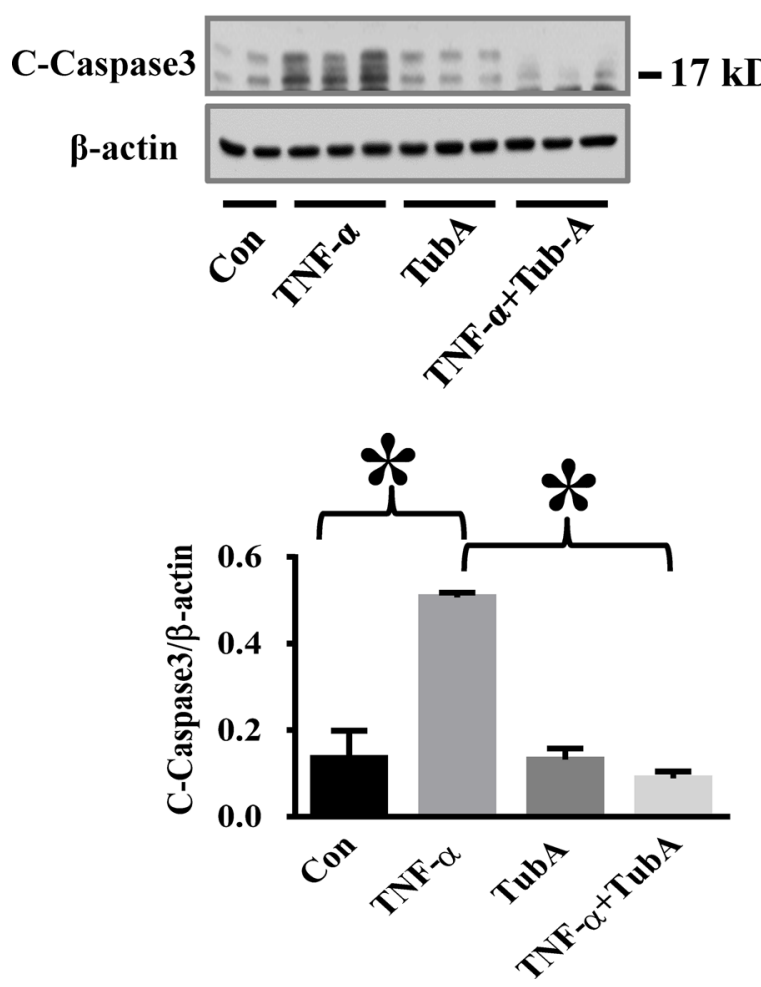

B
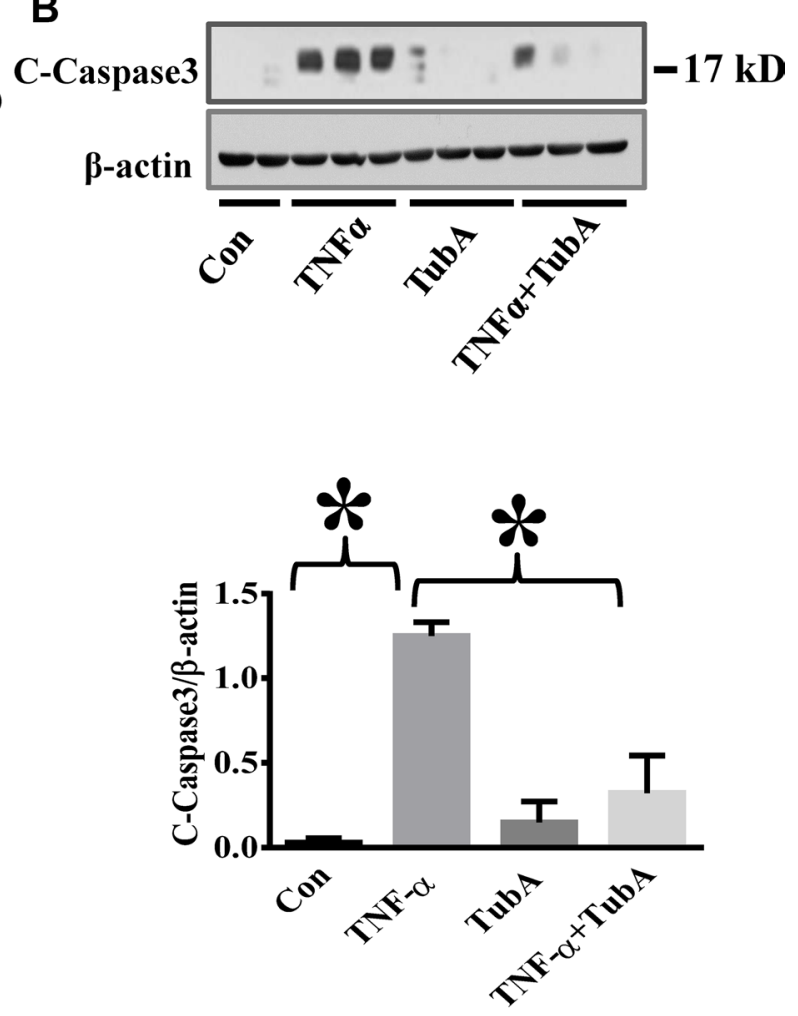

Figure 1: HDAC6 inhibition by Tubastatin A attenuates caspase-3 activation in endothelial cells. HPAECs and HLMVECs were pre-treated with Tubastatin A (TubA, $3 \mu \mathrm{M})$ for $6 \mathrm{~h}$, then challenged with TNF- $\alpha(20 \mathrm{ng} / \mathrm{ml})$ for $18 \mathrm{~h}$. Cells were divided into 4 groups: Control (Con), TNF- $\alpha$ alone (TNF $\alpha$ ), Tubastatin A alone (TubA), and TNF- $\alpha+$ Tubastatin A (TNF $\alpha+$ TubA). Representative blots and densitometry analysis of cleaved-caspase-3 in HPAECs (A) and HLMVECs (B). ${ }^{*} P<0.05$ versus TNF- $\alpha$ group, $n=3$. 
prevent TNF- $\alpha$-induced damage to endothelial cellcell junctions. We observed endothelial cells ZO-1 disassembly at tight junctions after TNF- $\alpha$ challenged by immunofluorescence assay. Pre-treatment with Tubstatin A prevented the disruption in HPAECs and HLMVECs (Figure 3).

\section{HDAC6 inhibition blocks endotoxin-induced caspase-3 activation and VE-Cadherin down- regulation in the lung}

To assess the therapeutic potential of HDAC6 inhibition against endothelial cell injury during acute inflammation, we examined the effects of CAY 10603 on caspase- 3 activation in a mouse model of endotoxemia. In our studies, endotoxin-induced caspase- 3 activation in the lung tissues was significantly inhibited by CAY 10603 and Tubastatin A pre-treatment. (Figure 4). Furthermore, endotoxin challenge caused down-regulation of adherens junction protein VE-Cadherin in the lung tissues. The down-regulation of VE-Cadherin was also blocked by CAY10603 and Tubastatin A pre-treatment (Figure 5).

\section{HDAC6 inhibition reduces TNF- $\alpha$-induced endothelial permeability and attenuates lung edema formation in endotoxemia}

TNF- $\alpha$ is a major pro-inflammatory mediator known to induce trans-endothelial hyper-permeability during inflammation $[1,7,9]$. We examined the effects of HDAC6 inhibition by CAY10603 on TNF- $\alpha$-induced endothelial permeability. TNF- $\alpha$ challenge caused increased endothelial permeability to FITC-dextran in HPAECs. Cells pre-treated with CAY10603 exhibited significant reduction of TNF- $\alpha$-induced endothelial permeability to FITC-dextran (Figure 6A). We also examined the effects of CAY10603 on lung vascular permeability in the mouse model of endotoxemia. In our studies, endotoxin-induced lung edema formation was significantly inhibited by CAY10603 pre-treatment (Figure 6B).
A

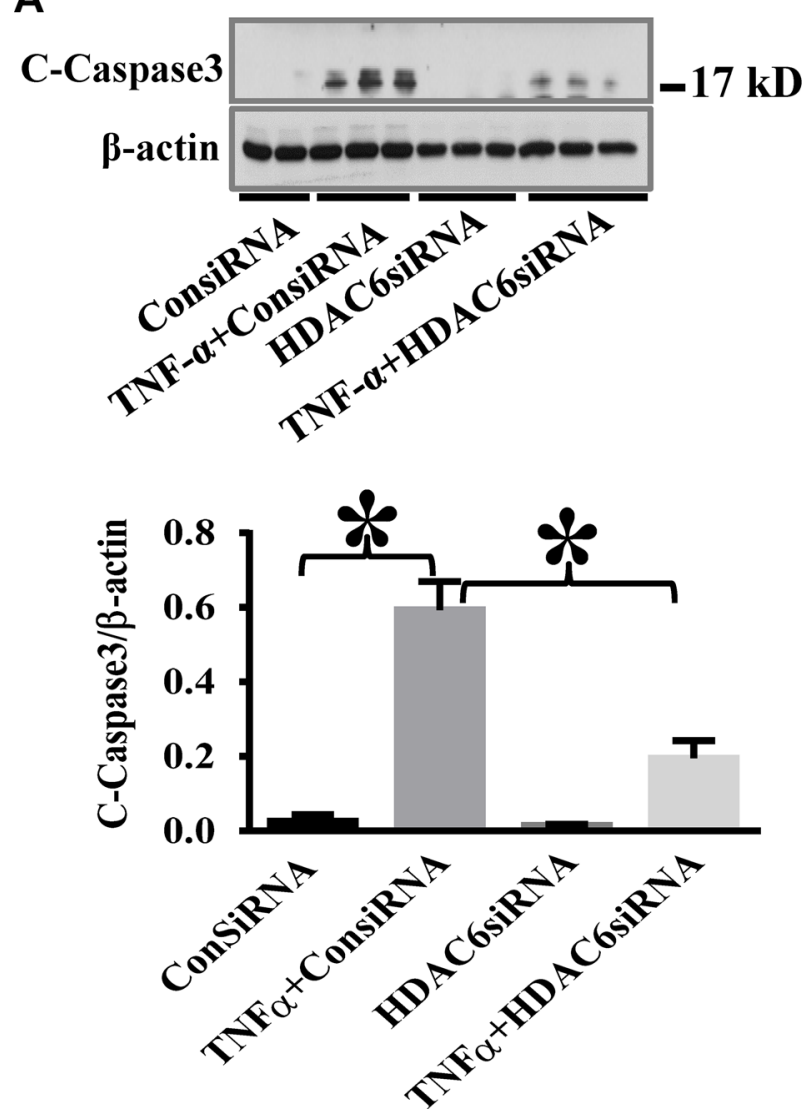

B
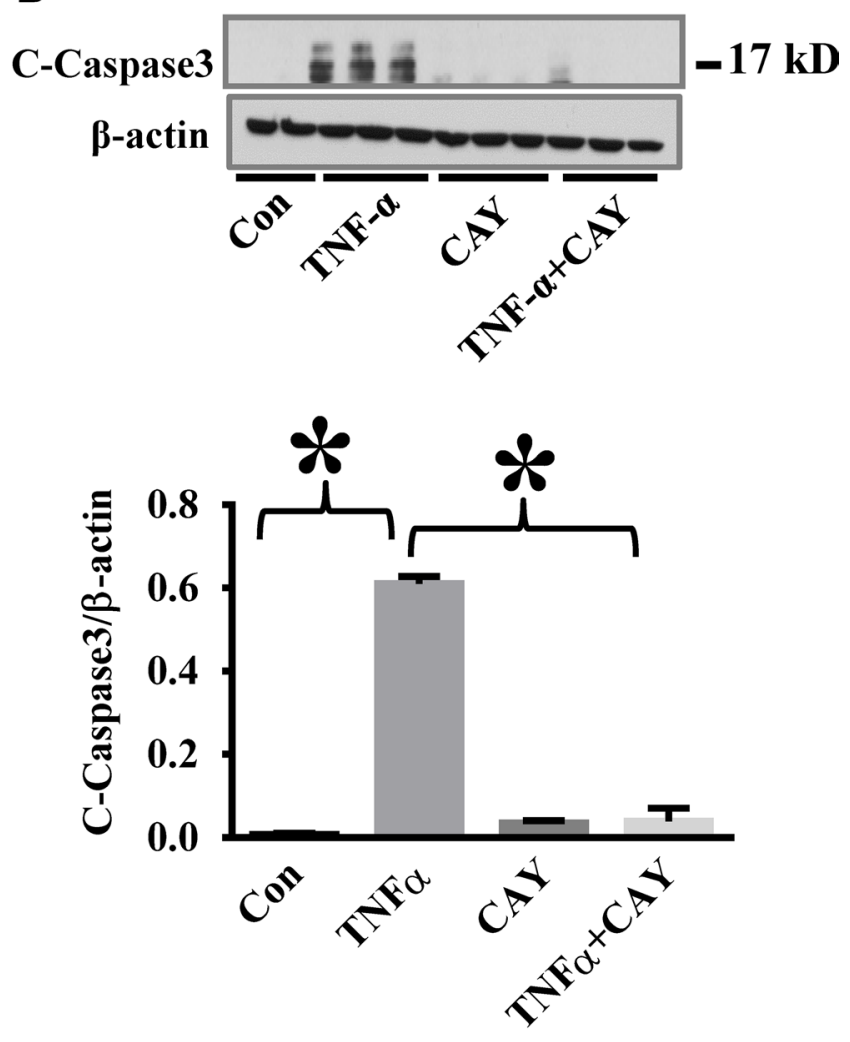

Figure 2: HDAC6 knockdown and HDAC6 inhibition by CAY10603 block TNF- $\alpha$-induced caspase-3 activation in endothelial cells. (A) HPAECs were transfected with HDAC6 siRNA or control siRNA for $48 \mathrm{~h}$, then challenged with TNF- $\alpha$ (20 ng/ml) for $24 \mathrm{~h}$. Cells were divided into 4 groups: Control (Con), TNF- $\alpha$ alone (TNF $\alpha$ ), siRNA alone (siRNA), and TNF- $\alpha+\operatorname{siRNA}(\mathrm{TNF} \alpha+\operatorname{siRNA}$ ). (B) HPAECs were pre-treated with CAY10603 (CAY, $0.1 \mu \mathrm{M})$ for $6 \mathrm{~h}$, then challenged with TNF $\alpha(20 \mathrm{ng} / \mathrm{ml})$ for $18 \mathrm{~h}$. Cells were divided into 4 groups: Control (Con), TNF- $\alpha$ alone (TNF $\alpha$ ), CAY10603 alone (CAY), and TNF- $\alpha+$ CAY10603 (TNF $\alpha+C A Y)$. Representative blots and densitometry analysis of cleaved caspase- $3 . * P<0.05$ versus TNF- $\alpha$ group, $n=3$. 
A

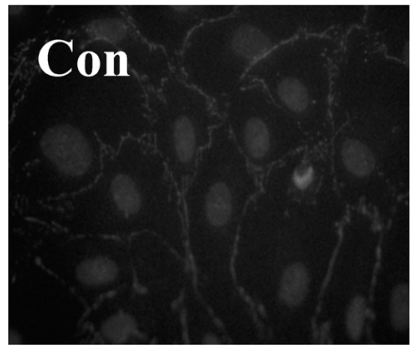

\section{TubA}

A
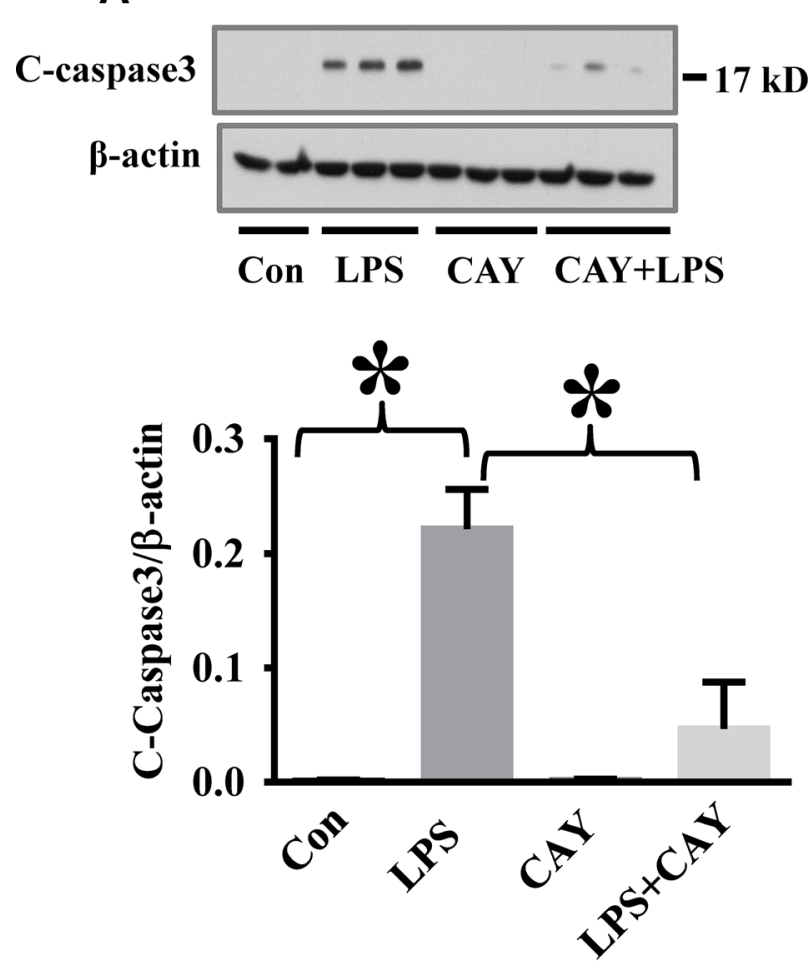

B
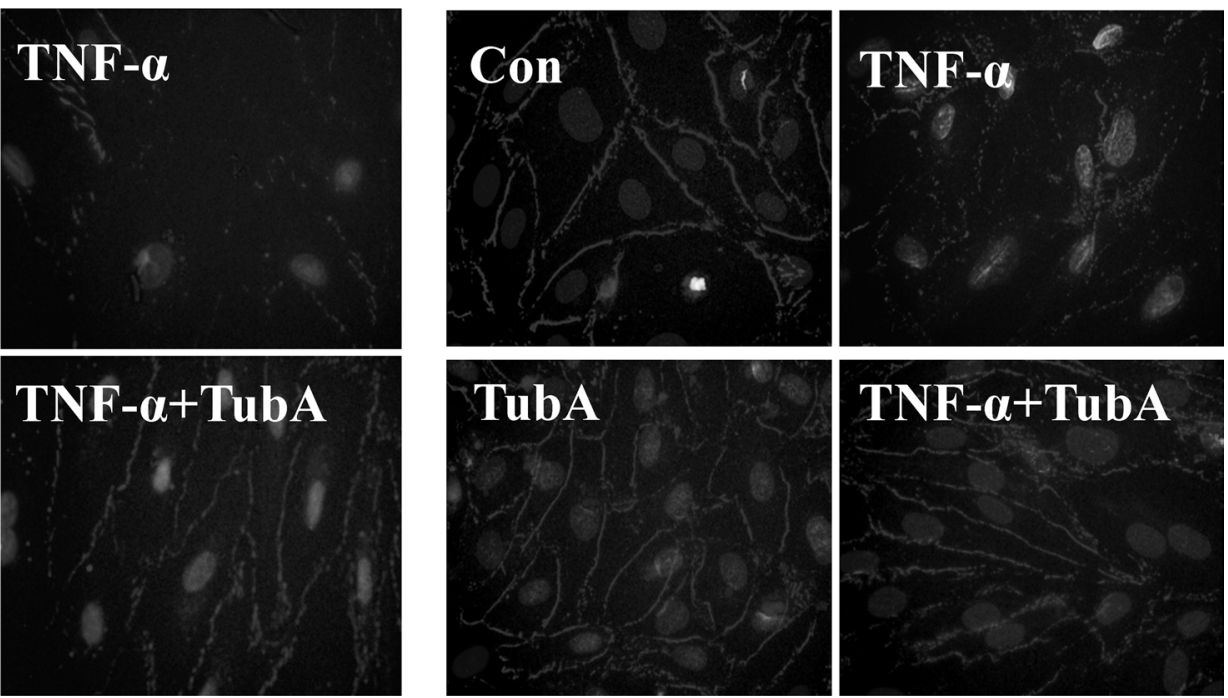

\section{TNF- $a+$ TubA}

Figure 3: HDAC6 inhibition prevents TNF- $\alpha$-induced ZO-1 disassembly in endothelial cells. HPAECs and HLMVECs were pre-treated with Tubastatin A (TubA, $3 \mu \mathrm{M})$ for $6 \mathrm{~h}$, then challenged with TNF- $\alpha(20 \mathrm{ng} / \mathrm{ml})$ for $18 \mathrm{~h}$. Cells were divided into 4 groups: Control (Con), TNF- $\alpha$ alone (TNF $\alpha$ ), Tubastatin A alone (TubA), and TNF- $\alpha+$ Tubastatin A (TNF $\alpha+$ TubA). Immunofluorescence staining of ZO-1 in HPAECs (A) and HLMVECs (B). Images are representatives of three to six independent experiments.

B
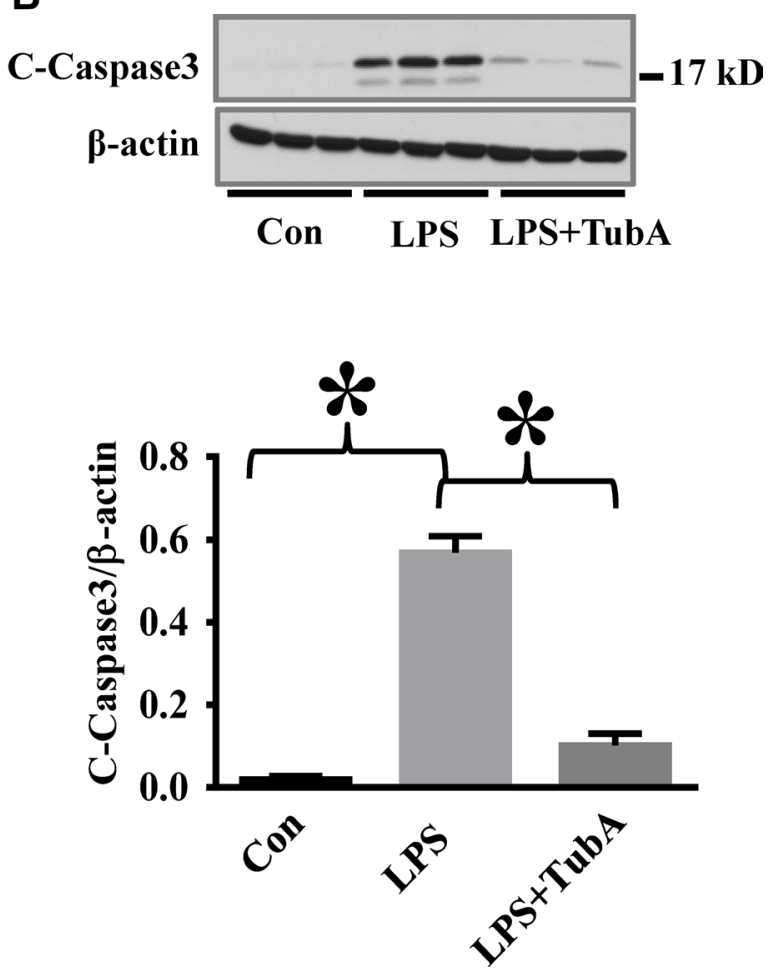

Figure 4: HDAC6 inhibition reduced LPS-induced lung caspase-3 activation in endotoxemia. (A) C57BL/6 mice were divided into four groups: Control (Con, $n=6$ ); LPS (LPS, $n=7$ ), CAY10603 (CAY, $n=6$ ); CAY10603+LPS (CAY+LPS, $n=7$ ). Mice were pre-treated with CAY10603 for $2 \mathrm{~h}$, then challenged with LPS for $24 \mathrm{~h}$. Lung tissue were collected. (B) C57/B6 mice were divided into three groups: Control (Con, $n=6)$; LPS $(n=7)$; Tubastatin A (TubA) + LPS $(n=6)$. Mice were pre-treated with Tubastatin A for $6 \mathrm{~h}$, then challenged with LPS. $24 \mathrm{~h}$ after LPS challenge, lung tissues were collected. Representative blots and densitometry analysis of cleaved caspase- 3 in lung tissues. ${ }^{*} P<0.05$ versus LPS group. 
A

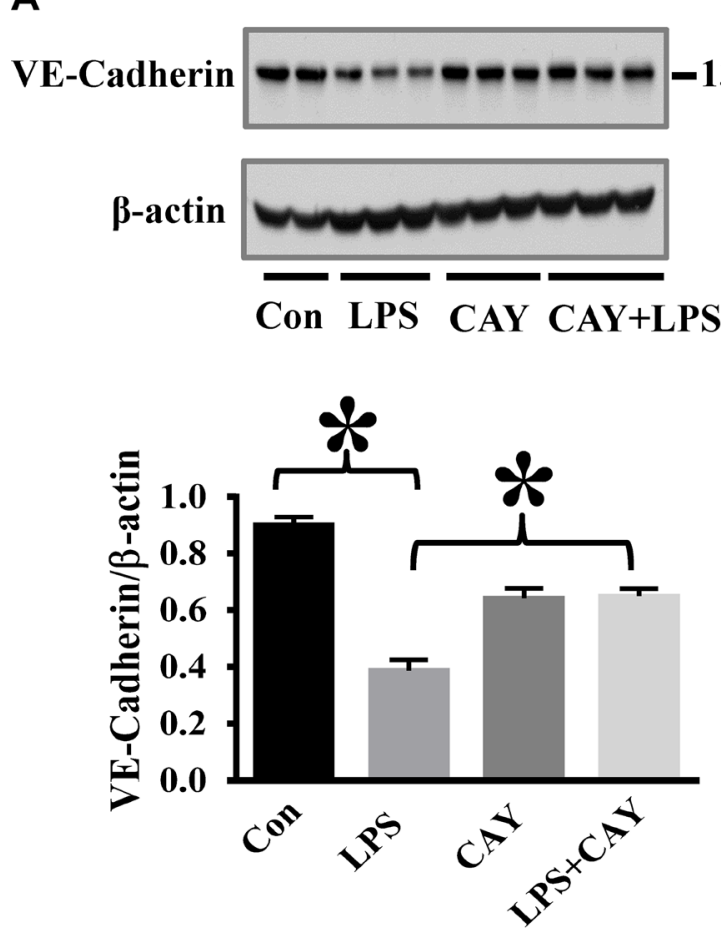

B

VE-Cadherin

B-actin
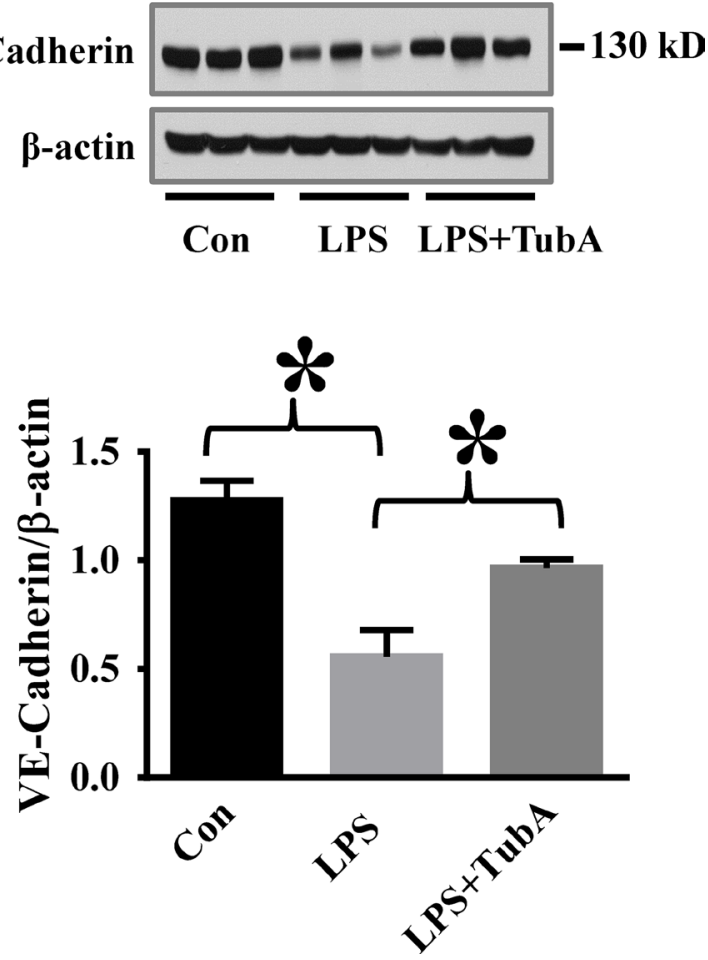

Figure 5: HDAC6 inhibition prevents LPS-induced VE-cadherin down-regulation in lung tissues. (A) C57BL/6 mice were divided into four groups: Control (Con, $n=6$ ); LPS (LPS, $n=7$ ), CAY 10603 (CAY, $n=6$ ); CAY10603+LPS (CAY+LPS, $n=7$ ). Mice were challenged by CAY 10603 for $2 \mathrm{~h}$, then challenged with LPS for $24 \mathrm{~h}$. Lung tissue were collected. (B) C57BL/6 mice were divided into three groups: Control (Con, $n=6)$; LPS $(n=7)$; Tubastatin A (TubA)+LPS $(n=6)$. Mice were challenged by LPS with or without Tubastatin A (TubA) pre-treatment. $24 \mathrm{~h}$ after LPS challenge, lung tissues were collected. Representative blots and densitometry analysis of VE-cadherin level in lung tissues. $* P<0.05$ versus LPS group.
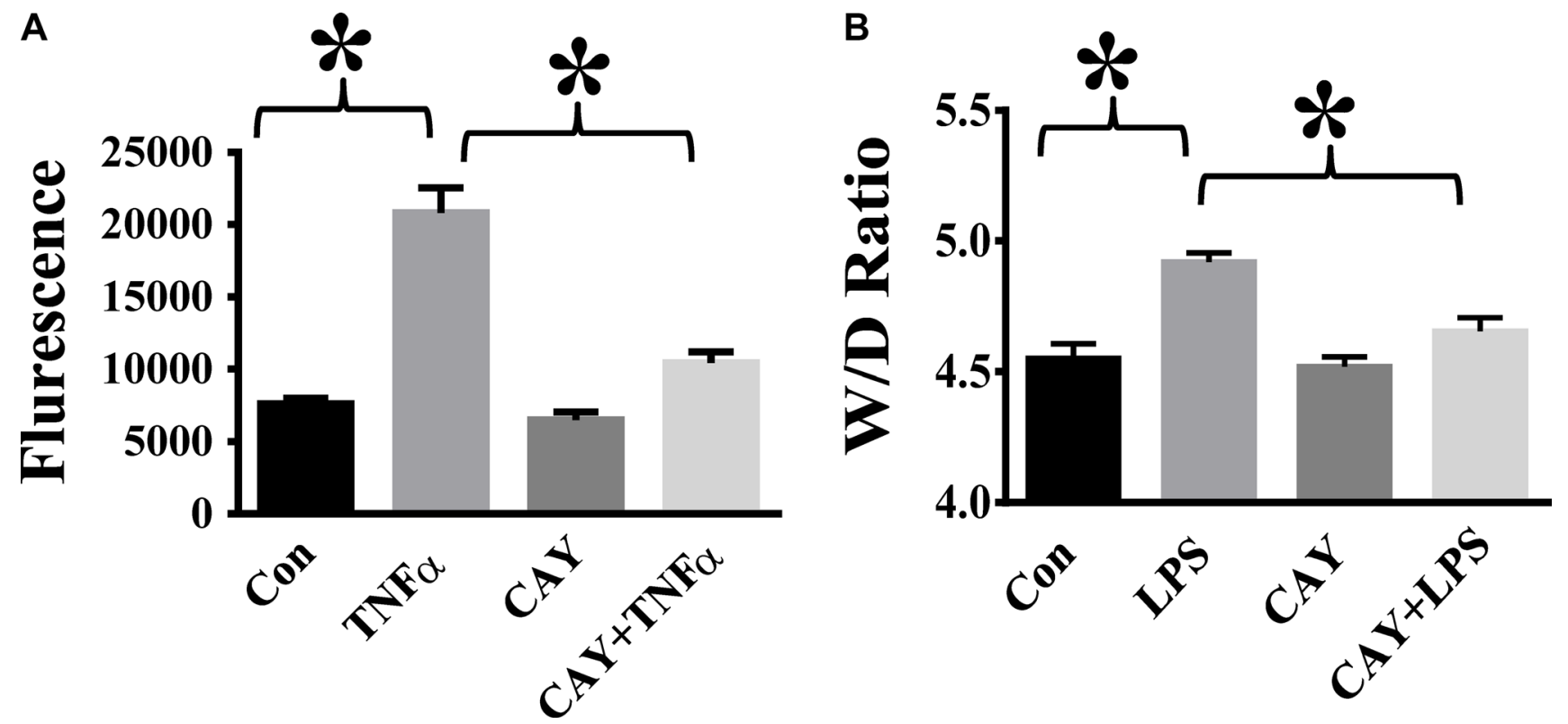

Figure 6: HDAC6 inhibition alleviates TNF- $\alpha$-induced endothelial hyper-permeability and lung edema in endotoxemia. (A) HPAECs were pre-treated with CAY $10603(\mathrm{CAY}, 0.1 \mu \mathrm{M})$ for $6 \mathrm{~h}$, then challenged with TNF $\alpha(20 \mathrm{ng} / \mathrm{ml})$ for $18 \mathrm{~h}$. Cells were divided into 4 groups: Control (Con), TNF- $\alpha$ alone (TNF $\alpha$ ), CAY10603 alone (CAY), and TNF- $\alpha+C A Y 10603$ (TNF $\alpha+C A Y)$. TNF- $\alpha$-induced endothelial permeability to FITC-Dextran was measured $(n=4)$. $* P<0.05$ versus TNF- $\alpha$ group. (B) C57BL/6 mice were divided into four groups: Control (Con, $n=6$ ); LPS (LPS, $n=7$ ), CAY 10603 (CAY, $n=6$ ); CAY 10603+LPS (CAY+LPS, $n=7$ ). Mice were pre-treated with CAY10603 for $2 \mathrm{~h}$, then challenged with LPS for $24 \mathrm{~h}$. Lung Wet/Dry weight ratio was measured. $* P<0.05$ versus LPS group. 


\section{DISCUSSION}

TNF- $\alpha$ is a potent pro-inflammatory mediator known to cause endothelial cell apoptosis and endothelial barrier disruption [7, 9, 31-33]. TNF- $\alpha$ plays an important role in many inflammatory diseases including lung vascular injury [1]. Activation of caspases including caspase 3 contributes to TNF- $\alpha$ induces endothelial cell dysfunction such as hyper-permeability [2-4]. Caspase activation mediates apoptotic signaling and disruption of cell junction complex [2-4], contributing to TNF- $\alpha$-induced breakdown of endothelial barrier function [2-4]. Similar effects were observed in our in vitro studies in primary human lung endothelial cells. Therefore, new agents that can inhibit TNF- $\alpha$-induced caspase activation and preserve endothelial cell junctions could serve as therapeutic agents against TNF- $\alpha$-induced endothelial cell dysfunction.

HDAC6 inhibition has been reported to prolong survival in murine models of systemic inflammation and injury $[20,24,25]$. However, mechanisms underlying the protective effects observed by HDAC6 inhibition remain to be determined. In the present study, we demonstrated that HDAC6 inhibition potently inhibited TNF- $\alpha$-induced caspase 3 activation and endothelial barrier dysfunction, suggesting that HDAC6 inhibition could provide protection against inflammation-mediated endothelial cell injury.

Caspase activation mediates apoptosis and redistribution of proteins that are known to modulate endothelial barrier function including $\mathrm{ZO}-1$ and VECadherin [2-4]. Protein acetylation can modulate caspase activation [2]. In our studies, HDAC6 inhibition by HDAC6 inhibitors or siRNA knockdown resulted in reduced caspase 3 activation. It has been reported that microtubule disruption leads to increased apoptotic signaling and caspase activation $[34,35] . \alpha$-tubulin is a endogenous substrate of HDAC6 [10, 11, 36-38]. Acetylation of $\alpha$-tubulin at Lys 40 stabilizes microtubule structures [12,38-41]. The decreased caspase 3 activation by HDAC6 inhibition could be due to its protection against microtubule disassembly by $\alpha$-tubulin acetylation. HDAC6 has been reported to modulate the function of chaperones such as Hsp90 [17]. Inhibition of HDAC6 down-regulates Hsp90-dependent RhoA activity and signaling, which then suppresses actin cytoskeleton reorganization and cell contraction [17]. HDAC6 has also been reported to modulate the function of Survivin, an anti-apoptotic protein [42]. Survivin inhibits apoptotic signaling by inactivating caspases [42]. Our data suggest that inhibition of caspase-3 activation by HDAC6 inhibition provides potent protection against TNF- $\alpha$ induced endothelial cell dysfunction.

We also showed that HDAC6 inhibition effectively suppress caspase- 3 activation and maintain lung endothelial barrier integrity in vivo. HDAC6 inhibitors prevented caspase 3 activation in lung tissues with reduced lung edema in a mouse model of endotoxemia. Maintaining VE-
Cadherin expression is critical in supporting lung vascular barrier integrity [43]. We demonstrated that Tubastatin A and CAY 10603 were able to prevent the reduction of VECadherin in lung tissues after LPS challenge. Our results suggest that HDAC6 inhibition is a new approach that can modulate endothelial cell junction stability in inflammatory lung injury. More studies are needed in the future to further investigate this new pathway.

In summary, selective HDAC6 inhibition by CAY10603 and Tubastatin A prevents TNF- $\alpha$-induced endothelial cell dysfunction. HDAC6 inhibitors prevent caspase-3 activation in endothelial cells and inhibit TNF$\alpha$-induced endothelial barrier dysfunction by maintaining cell-cell junction integrity. HDAC6 inhibitors prevented endotoxin-induced lung caspase- 3 activation and lung edema, suggesting that selective HDAC6 inhibition possesses therapeutic potential to treat endothelial cell dysfunction during acute inflammation.

\section{MATERIALS AND METHODS}

\section{Reagents}

$\beta$-actin, ZO-1, caspase-3 antibodies were purchased from Cell Signaling Technology (Danvers, Massachusetts). VE-cadherin antibody was obtained from Santa Cruz Biotechnology (Dallas, Texas). Recombinant Human TNF- $\alpha$ was from R\&D Systems (Minneapolis, Minnesota). Tubastatin A, CAY10603 were obtained from Selleck Chemicals (Houston, Teaxs). Lipopolysaccharide (LPS) from Escherichia coli 0111:B4 was purchased from Sigma Aldrich (St. Louis, Missouri). Endothelial growth medium (EBM-2) was obtained from Lonza (Allendale, NJ). In Vitro Vascular Permeability Assay (96-Well) kit was purchased from EMD Millipore (Billerica, Massachusetts). Lipofectamine RNAiMAX reagent, HDAC6 siRNA and control siRNA were obtained from Life technologies (Carlsbad, California).

\section{Cell culture}

Human pulmonary arterial endothelial cells (HPAECs) and human lung microvascular endothelial cells (HLMVECs) were purchased from Lonza (Allendale, NJ). Cells were grown in EGM-2 supplemented with fetal bovine serum (FBS) and cultured in an incubator at $37^{\circ} \mathrm{C}$ in $5 \% \mathrm{CO} 2$ and $95 \%$ air. Cells from passages 5 to 9 were used in the experiments.

\section{HDAC6 siRNA knockdown}

HDAC6 siRNA knockdown was conducted as described previously [41]. Cells were transfected with siRNAs for $48 \mathrm{~h}$ according to the manufacture's protocol. siRNA-transfected cells were then stimulated with $20 \mathrm{ng} / \mathrm{ml}$ TNF- $\alpha$ for $24 \mathrm{~h}$. 


\section{Immunofluorescence and immunoblotting assays}

For immunofluorescence assay, cells were grown on glass coverslips pre-coated with $0.1 \%$ gelation. After the treatment, cells were fixed in $-20^{\circ} \mathrm{C}$ methanol and washed with PBS on ice. Cells were blocked with $1 \%$ BSA in PBS, then incubated with primary antibody. After 3 washes with TBS, cells were incubated with Alexa Fluor 594 secondary antibody. The coverslips were mounted on the slides with DAPI. Immunoblotting assays of cell and lung tissue samples were conducted as described previously [43, 44].

\section{Mouse model of endotoxemia}

Eleven to twelve week-old male C57BL/6 mice were purchased from Jackson Lab. All experiments and animal care procedures were approved by the Institutional Animal Care and Use Committee of the University of Kentucky. Endotoxemia was induced by I.P. injection of $7.5 \mathrm{mg} / \mathrm{kg}$ LPS in phosphate buffered saline (PBS). Mice were pre-treated by I.P. injection with Tubstatin A $(9 \mathrm{mg} / \mathrm{kg}$ body weight) for $6 \mathrm{~h}$ or CAY10603 $(5 \mathrm{mg} / \mathrm{kg}$ body weight) for $2 \mathrm{~h}$ before LPS challenge. Experiments were terminated $24 \mathrm{~h}$ after LPS challenge. Lung wet/dry weight ratio was assessed as described previously [43].

\section{Endothelial cell permeability assay}

In Vitro Vascular Permeability Assay (96-Well) kit was used to measure endothelial cell monolayer permeability to FITC-dextran according to the manufacture's protocol. Briefly, endothelial cells were seeded into the insert. $72 \mathrm{~h}$ later, endothelial cells formed a monolayer. Endothelial cell monolayers were pretreated with CAY10603 (CAY, $0.1 \mu \mathrm{M})$ for $6 \mathrm{~h}$, and then stimulated with TNF- $\alpha$ for $18 \mathrm{~h}$. A high molecular weight $(2000 \mathrm{kD})$ FITC-Dextran was diluted in EGM-2 medium (1:40) and added into the insert. 20 minutes after the incubation, $100 \mu 1$ medium from lower chamber was collected. Fluorescence intensity was measured in a plate with a fluorescence plate reader.

\section{Statistical analysis}

Results are expressed as means $\pm \mathrm{SE}$ of 3 to 6 independent experiments. ANOVA and post hoc multiple comparison tests were used for multiple groups. The Student's $t$-test was used for comparisons of two groups. Statistical significance was assigned to $P$ values $<0.05$.

\section{ACKNOWLEDGMENTS AND FUNDING}

Jinyan $\mathrm{Yu}$ is a recipient of the State Scholarship from China Scholarship Council.

\section{CONFLICTS OF INTEREST}

All the authors declared no competing interests.

\section{REFERENCES}

1. Johnson ER, Matthay MA. Acute lung injury: epidemiology, pathogenesis, and treatment. J Aerosol Med Pulm Drug Deliv. 2010; 23:243-252.

2. Shi M, Lu XJ, Zhang J, Diao H, Li G, Xu L, Wang T, Wei J, Meng W, Ma JL, Yu H, Wang YG. Oridonin, a novel lysine acetyltransferases inhibitor, inhibits proliferation and induces apoptosis in gastric cancer cells through p53and caspase-3-mediated mechanisms. Oncotarget. 2016; 7:22623-31. doi: 10.18632/oncotarget.8033.

3. Sawant DA, Tharakan B, Tobin RP, Reilly J, Hunter FA, Newell MK, Smythe WR, Childs EW. Microvascular endothelial cell hyperpermeability induced by endogenous caspase 3 activator staurosporine. J Trauma Acute Care Surg. 2013; 74:516-523.

4. Zehendner CM, Librizzi L, de Curtis M, Kuhlmann CR, Luhmann HJ. Caspase-3 contributes to ZO-1 and Cl-5 tight-junction disruption in rapid anoxic neurovascular unit damage. PLoS One. 2011; 6:e16760.

5. Rodrigues SF, Granger DN. Blood cells and endothelial barrier function. Tissue Barriers. 2015; 3:e978720.

6. Petrache I, Birukova A, Ramirez SI, Garcia JG, Verin AD. The role of the microtubules in tumor necrosis factor-alphainduced endothelial cell permeability. Am J Respir Cell Mol Biol. 2003; 28:574-581.

7. Goldblum SE, Ding X, Campbell-Washington J. TNF-alpha induces endothelial cell F-actin depolymerization, new actin synthesis, and barrier dysfunction. Am J Physiol. 1993; 264:C894-905.

8. Yang J, Yao W, Qian G, Wei Z, Wu G, Wang G. Rab5mediated VE-cadherin internalization regulates the barrier function of the lung microvascular endothelium. Cell Mol Life Sci. 2015.

9. Marcos-Ramiro B, Garcia-Weber D, Millan J. TNF-induced endothelial barrier disruption: beyond actin and Rho. Thromb Haemost. 2014; 112:1088-1102.

10. Iaconelli J, Huang JH, Berkovitch SS, Chattopadhyay S, Mazitschek R, Schreiber SL, Haggarty SJ, Karmacharya R. HDAC6 inhibitors modulate Lys49 acetylation and membrane localization of beta-catenin in human iPSCderived neuronal cells. ACS Chem Biol. 2015; 10:883-890.

11. Li Y, Zhang X, Polakiewicz RD, Yao TP, Comb MJ. HDAC6 is required for epidermal growth factor-induced beta-catenin nuclear localization. J Biol Chem. 2008; 283:12686-12690.

12. Zhang Y, Li N, Caron C, Matthias G, Hess D, Khochbin S, Matthias P. HDAC-6 interacts with and deacetylates tubulin and microtubules in vivo. EMBO J. 2003; 22:1168-1179.

13. Woan KV, Lienlaf M, Perez-Villaroel P, Lee C, Cheng F, Knox T, Woods DM, Barrios K, Powers J, Sahakian E, 
Wang HW, Canales J, Marante D, et al. Targeting histone deacetylase 6 mediates a dual anti-melanoma effect: Enhanced antitumor immunity and impaired cell proliferation. Mol Oncol. 2015; 9:1447-1457.

14. Soo Youn G, Ju SM, Choi SY, Park J. HDAC6 mediates HIV-1 tat-induced proinflammatory responses by regulating MAPK-NF-kappaB/AP-1 pathways in astrocytes. Glia. 2015.

15. Yan B, Xie S, Liu Z, Ran J, Li Y, Wang J, Yang Y, Zhou J, Li D, Liu M. HDAC6 deacetylase activity is critical for lipopolysaccharide-induced activation of macrophages. PLoS One. 2014; 9:e110718.

16. Spange S, Wagner T, Heinzel T, Kramer OH. Acetylation of non-histone proteins modulates cellular signalling at multiple levels. Int J Biochem Cell Biol. 2009; 41:185-198.

17. Joshi AD, Barabutis N, Birmpas C, Dimitropoulou C, Thangjam G, Cherian-Shaw M, Dennison J, Catravas JD. Histone deacetylase inhibitors prevent pulmonary endothelial hyperpermeability and acute lung injury by regulating heat shock protein 90 function. Am J Physiol Lung Cell Mol Physiol. 2015; 309:L1410-1419.

18. Chattopadhyay S, Fensterl V, Zhang Y, Veleeparambil M, Wetzel JL, Sen GC. Inhibition of viral pathogenesis and promotion of the septic shock response to bacterial infection by IRF-3 are regulated by the acetylation and phosphorylation of its coactivators. MBio. 2013; 4.

19. Butler KV, Kalin J, Brochier C, Vistoli G, Langley B, Kozikowski AP. Rational design and simple chemistry yield a superior, neuroprotective HDAC6 inhibitor, tubastatin A. J Am Chem Soc. 2010; 132:10842-10846.

20. Chang Z, Li Y, He W, Liu B, Halaweish I, Bambakidis T, Liang Y, Alam HB. Selective inhibition of histone deacetylase 6 promotes survival in a rat model of hemorrhagic shock. J Trauma Acute Care Surg. 2015; 79:905-910.

21. Choi SY, Ryu Y, Kee HJ, Cho SN, Kim GR, Cho JY, Kim HS, Kim IK, Jeong MH. Tubastatin A suppresses renal fibrosis via regulation of epigenetic histone modification and Smad3-dependent fibrotic genes. Vascul Pharmacol. 2015; 72:130-140.

22. Lee J, Hong EC, Jeong H, Hwang JW, Kim H, Bae EK, Ahn JK, Choi YL, Han J, Cha HS, Koh EM. A novel histone deacetylase 6-selective inhibitor suppresses synovial inflammation and joint destruction in a collagen antibodyinduced arthritis mouse model. Int J Rheum Dis. 2015; 18:514-523.

23. Vishwakarma S, Iyer LR, Muley M, Singh PK, Shastry A, Saxena A, Kulathingal J, Vijaykanth G, Raghul J, Rajesh N, Rathinasamy S, Kachhadia V, Kilambi N, et al. Tubastatin, a selective histone deacetylase 6 inhibitor shows anti-inflammatory and anti-rheumatic effects. Int Immunopharmacol. 2013; 16:72-78.

24. Cheng X, Liu Z, Liu B, Zhao T, Li Y, Alam HB. Selective histone deacetylase 6 inhibition prolongs survival in a lethal two-hit model. J Surg Res. 2015; 197:39-44.
25. Li Y, Zhao T, Liu B, Halaweish I, Mazitschek R, Duan X, Alam HB. Inhibition of histone deacetylase 6 improves long-term survival in a lethal septic model. J Trauma Acute Care Surg. 2015; 78:378-385.

26. Zhao T, Li Y, Bronson RT, Liu B, Velmahos GC, Alam HB. Selective histone deacetylase-6 inhibition attenuates stress responses and prevents immune organ atrophy in a lethal septic model. Surgery. 2014; 156:235-242.

27. Zhao T, Li Y, Liu B, Halaweish I, Mazitschek R, Alam HB. Selective inhibition of histone deacetylase 6 alters the composition of circulating blood cells in a lethal septic model. J Surg Res. 2014; 190:647-654.

28. Zhao T, Li Y, Liu B, Pan B, Cheng X, Georgoff P, Alam HB. Inhibition of histone deacetylase 6 restores innate immune cells in the bone marrow in a lethal septic model. J Trauma Acute Care Surg. 2016; 80:34-41.

29. Lei M, Bai X, Yang T, Lai X, Qiu W, Yang L, Lian X. Gsdma3 is a new factor needed for TNF-alpha-mediated apoptosis signal pathway in mouse skin keratinocytes. Histochem Cell Biol. 2012; 138:385-396.

30. Wang DH, Hu JR, Wang LY, Hu YJ, Tan FQ, Zhou H, Shao JZ, Yang WX. The apoptotic function analysis of p53, Apaf1, Caspase 3 and Caspase 7 during the spermatogenesis of the Chinese fire-bellied newt Cynops orientalis. PLoS One. 2012; 7:e39920.

31. Prasain N, Stevens T. The actin cytoskeleton in endothelial cell phenotypes. Microvasc Res. 2009; 77:53-63.

32. Bogatcheva NV, Verin AD. The role of cytoskeleton in the regulation of vascular endothelial barrier function. Microvasc Res. 2008; 76:202-207.

33. Acquavella N, Quiroga MF, Wittig O, Cardier JE. Effect of simvastatin on endothelial cell apoptosis mediated by Fas and TNF-alpha. Cytokine. 2010; 49:45-50.

34. Carew JS, Esquivel JA, 2nd, Espitia CM, Schultes CM, Mulbaier M, Lewis JD, Janssen B, Giles FJ, Nawrocki ST. ELR510444 inhibits tumor growth and angiogenesis by abrogating HIF activity and disrupting microtubules in renal cell carcinoma. PLoS One. 2012; 7:e31120.

35. Cheng YC, Liou JP, Kuo CC, Lai WY, Shih KH, Chang CY, Pan WY, Tseng JT, Chang JY. MPT0B098, a novel microtubule inhibitor that destabilizes the hypoxiainducible factor-1alpha mRNA through decreasing nuclearcytoplasmic translocation of RNA-binding protein HuR. Mol Cancer Ther. 2013; 12:1202-1212.

36. Bobrowska A, Paganetti P, Matthias P, Bates GP. Hdac6 knock-out increases tubulin acetylation but does not modify disease progression in the R6/2 mouse model of Huntington's disease. PLoS One. 2011; 6:e20696.

37. Hubbert C, Guardiola A, Shao R, Kawaguchi Y, Ito A, Nixon A, Yoshida M, Wang XF, Yao TP. HDAC6 is a microtubuleassociated deacetylase. Nature. 2002; 417:455-458.

38. Matsuyama A, Shimazu T, Sumida Y, Saito A, Yoshimatsu Y, Seigneurin-Berny D, Osada H, Komatsu Y, Nishino N, Khochbin S, Horinouchi S, Yoshida M. In vivo 
destabilization of dynamic microtubules by HDAC6mediated deacetylation. EMBO J. 2002; 21:6820-6831.

39. Tran AD, Marmo TP, Salam AA, Che S, Finkelstein E, Kabarriti R, Xenias HS, Mazitschek R, Hubbert C, Kawaguchi Y, Sheetz MP, Yao TP, Bulinski JC. HDAC6 deacetylation of tubulin modulates dynamics of cellular adhesions. J Cell Sci. 2007; 120:1469-1479.

40. Zilberman Y, Ballestrem C, Carramusa L, Mazitschek R, Khochbin S, Bershadsky A. Regulation of microtubule dynamics by inhibition of the tubulin deacetylase HDAC6. J Cell Sci. 2009; 122:3531-3541.

41. Yu J, Ma Z, Shetty S, Ma M, Fu J. Selective HDAC6 inhibition prevents TNF-alpha-induced lung endothelial cell barrier disruption and endotoxin-induced pulmonary edema.
Am J Physiol Lung Cell Mol Physiol. 2016; 311:L39-47. doi: 10.1152/ajplung.00051.2016.

42. Riolo MT, Cooper ZA, Holloway MP, Cheng Y, Bianchi C, Yakirevich E, Ma L, Chin YE, Altura RA. Histone deacetylase 6 (HDAC6) deacetylates survivin for its nuclear export in breast cancer. J Biol Chem. 2012; 287:10885-10893.

43. Gao R, Ma Z, Hu Y, Chen J, Shetty S, Fu J. Sirt1 restrains lung inflammasome activation in a murine model of sepsis. Am J Physiol Lung Cell Mol Physiol. 2015; 308:L847-853.

44. Fu J, Naren AP, Gao X, Ahmmed GU, Malik AB. Proteaseactivated receptor-1 activation of endothelial cells induces protein kinase Calpha-dependent phosphorylation of syntaxin 4 and Munc18c: role in signaling p-selectin expression. J Biol Chem. 2005; 280:3178-3184. 